\title{
Short scales were as effective as long scales in screening for depression in older patients
}

Pomeroy IM, Clark CR, Philp I. The effectiveness of very short scales for depression screening in elderly medical patients. Int J Geriatr Psychiatr 2001 Mar;16:321-6.

\section{QUESTION: In older patients, are short scales as effective as long scales in screening for depression?}

\section{Design}

Blinded comparison of 3 Geriatric Depression Scales (GDS-4, GDS-15, and GDS-30) and the Mental Health Inventory-1 (MHI-1) scale with diagnostic criteria of research of International Classification of Diseases10th edition (DCR-10).

\section{Setting}

A teaching hospital in the UK

\section{Patients}

87 patients (mean age $79 \mathrm{y}, 60 \%$ women) who were $>60$ years of age and attended the day rehabilitation facility or were admitted to the medical rehabilitation wards. Patients were excluded if they had an illness, communication problems, or a score of $<6$ on the 10-item Abbreviated Mental Test (AMT).

\section{Description of tests and diagnostic standard}

The GDS-30, GDS-4, 10-item AMT, and 5-item MHI were administered within 48 hours after an initial interview. Data for GDS-15 and MHI-1 were extracted from GDS-30 and MHI-5. Established cut points for diagnosis of depression were used for GDS-30, GDS-15, and GDS-4. The cut point for MHI-1 was set retrospectively. The clinical interview assessed mood and depression by using the DCR-10 (diagnostic standard).

\section{Main outcome measures}

Sensitivity, specificity, and area under the receiver operating characteristic (ROC) curve.

\section{Main results}

17 of the 87 patients (20\%) were diagnosed with depression by using the DCR-10. Sensitivity, specificity, positive and negative likelihood ratios, and ROC curve results for all tests are in the table. The 4 tests did not differ for screening of depression.

\section{Conclusion}

Short scales (Geriatric Depression Scale-4 and Mental Health Inventory-1) were comparable in sensitivity and specificity to long scales (Geriatric Depression Scale-30 and Geriatric Depression Scale-15) in screening for depression in older patients.

Source of funding: not stated.

For correspondence: Professor I Philp, Sheffield Institute for Studies on Ageing, Northern General Hospital, Herries Road, Sheffield S5 7AU, UK.

Operating characteristics of short and long scales to screen for depression in older patients*

\begin{tabular}{lcllll} 
Scales & Sensitivity & Specificity & +LR & -LR & $\begin{array}{l}\text { Area under ROC } \\
\text { curve }(95 \% \text { Cl) }\end{array}$ \\
MHI-1 & $88 \%$ & $71 \%$ & 3.08 & 0.17 & $0.88(0.79$ to 0.97$)$ \\
\hline GDS-4 & $82 \%$ & $67 \%$ & 2.50 & 0.26 & $0.80(0.68$ to 0.93$)$ \\
\hline GDS-15 & $82 \%$ & $60 \%$ & 2.06 & 0.29 & $0.82(0.71$ to 0.93$)$ \\
\hline GDS-30 & $100 \%$ & $62 \%$ & 2.70 & 0.00 & $0.85(0.77$ to 0.94$)$ \\
\hline
\end{tabular}

${ }^{*}$ GDS = Geriatric Depression Scale; $\mathrm{MHI}=$ Mental Health Inventory; ROC = receiver operating characteristic. Other diagnostic terms defined in glossary; LRs calculated from data in article.

\section{COMMENTARY}

Depression is common, serious, and treatable, but it is under-recognised, particularly in elderly people. Societal and cultural biases often hinder the diagnosis. ${ }^{1}$ The study by Pomeroy et al compares 4 different screening instruments of variable length and content. The authors found that all 4 screening instruments had similar accuracy for detecting depression, and the 1-item MHI-1 had the best combination of sensitivity and specificity.

These results need further validation for 3 reasons: first, this study assessed a small inpatient sample; second, fewer than half of the patients approached were included in the study; and third, a relatively low interrater reliability $(\kappa=0.40)$ existed for MHI-1. Furthermore, the cut point for the MHI was defined retrospectively, and the item itself was not done independently and was extracted from a longer scale.

Should the clinician wait for further validation before implementing this approach to screening for depression? The answer is a resounding "no"! The literature on screening for depression in general medical outpatients ${ }^{2}$ suggests that all of the screening instruments are relatively comparable, with sensitivity and specificity ranging from $80 \%$ to $90 \%$ : similar to the findings in this study. In a typical setting, a positive test result might raise the probability of depression from $10 \%$ to $15 \%$ to $35 \%$ to $45 \%$, and a negative test result might lower the probability of depression to $<5 \%$. Ensuring that all patients are screened for depression regularly is more important than small changes in the precision of the screening instrument. ${ }^{3}$

Scott Sherman, MD, MPH VA Center for the Study of Healthcare Provider Behavior Sepulveda, California, USA

1 Harman JS, Reynolds CF. Removing the barriers to effective depression treatment in old age. JAm Geriatr Soc 2000;48:1012-3.

2 Whooley MA, Avins AL, Miranda J, Browner WS. Case-finding instruments for depression: two questions are as good as many. J Gen Intern Med 1997;12:439-45.

3 Solberg LI, Korsen N, Oxman TE, Fischer LR, Bartels S. The need for a system in the care of depression. J Fam Pract 1999;48:973-9. 